\title{
«Wir wollen die Medizin kritisch reflektieren»
}

\section{Daniel Lüthi}

Freier Journalist und Fotograf, Medientrainer, Bern

Anatomie und Medizingeschichte sind in diesem historischen Gebäude vereint. Der denkmalgeschützte Hörsaal, weitgehend aus Holz, entstand um 1900 - ein Bijou. Vor etwa 40 Jahren wäre es beinahe dem Fortschrittsglauben zum Opfer gefallen. «Es ist ein eigentliches Theater, ausgerichtet auf die Wandtafel», erklärt Hubert Steinke.

\section{Zunehmende Spezialisierung}

Dem damaligen Chef der Anatomie sei es wichtig gewesen, bei den Studenten das Verständnis für den Kör- per zeichnend zu gewinnen. Wenn hier eine Leiche vorgeführt wurde, sei dies eine Inszenierung, ein kleines Spektakel gewesen, sagt Steinke, und es ist, als ob er langsam in seine Rolle als Dozent schlüpfen würde. Wobei etwas als besonders angenehm auffällt: Er ist einer, der so spricht, dass man ihn versteht. «Übrigens», fügt er hinzu: «Die Leichen wurden aus dem Raum hierhergebracht, wo heute mein Büro ist.»

Die Anatomie sei früher die Basis des gesamten Medizinstudiums gewesen, «sie hatte einen riesigen Stellenwert. Aber damals kannte man die Zellen noch nicht. Man ging von dem aus, was man sah.» Zunehmend

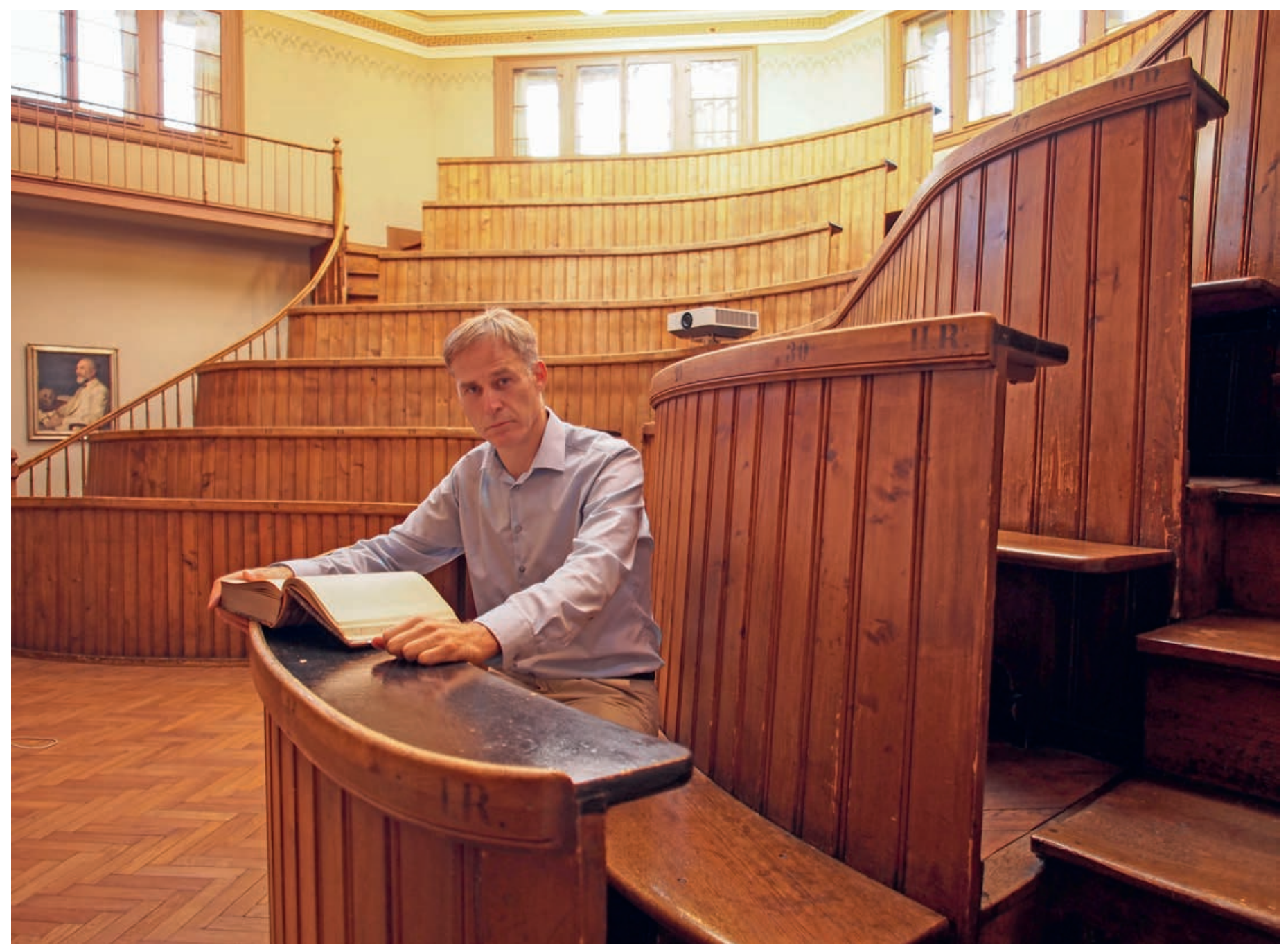


habe sich die Medizin dann von der makroskopischen auf die mikroskopische Ebene verlagert: eine der grossen Entwicklungen in der Medizin. Eine andere: die immer stärkere Spezialisierung und Fragmentierung der einzelnen Fächer. «Die Diskussion darüber, ob das eine positive Entwicklung ist oder nicht, und das Bedauern, dass die Einheit der Medizin immer mehr verloren geht - diese Diskussion gab's schon vor hundert Jahren. Wir müssen halt herausfinden, wie wir den Patienten trotzdem als Ganzes im Auge behalten können.» Tumor- oder Alterszentren beispielsweise seien Formen, die Medizin nicht nach Organen oder Fachrichtungen aufzuteilen, sondern einen ganzheitlicheren Ansatz zu verwirklichen.

"In unserer Gesellschaft ist eine starke Tendenz zu einer Medikalisierung zu beobachten.»

Entwicklungen aufzuzeigen und dazu die richtigen Fragen zu stellen: Das ist laut Hubert Steinke eine der zentralen Aufgaben seines Fachs. "Wir Medizinhistoriker wollen Geschichten aus der Geschichte erzählen und wir wollen Dinge erklären. Dabei haben wir vor allem ein Ziel: Wir wollen die Medizin kritisch reflektieren.»

Zu ihrer Technisierung beispielsweise sagt er, bei allem Respekt für das, was sie an positiven Errungenschaften gebracht hat: «Die Technik hat sich zunehmend zwischen Arzt und Patient gestellt.»

\section{Das gescheiterte Museum}

Einer der Orte, wo eine kritische Reflexion ausserhalb des Hörsaals, auch für die Öffentlichkeit, hätte möglich gemacht werden sollen, wäre das Medizinmuseum Bern gewesen. Hubert Steinke war designierter Direktor und er hatte, zusammen mit seinem Team, bereits sehr viel Energie und Leidenschaft in das Projekt gesteckt. Rund 10000 Objekte aus der Sammlung der Inselstiftung und jener des Medizinhistorischen Instituts standen bereit, in Zusammenarbeit mit dem Universitätsspital wurde während Jahren ein Konzept entwickelt, im Oktober hätte dieses erste solche Museum der Schweiz eröffnet werden sollen. Es hätte sich thematisch vor allem an Verben orientiert: forschen, schneiden, liegen, warten, messen. Einmal mehr rückte ein anderes Verb in den Vordergrund: kosten. Praktisch in letzter Minute kam von der neuen Direktion der Inselgruppe nämlich das überraschende «Nein» - aus finanziellen Gründen, wie es hiess. "Natürlich hat mich diese Absage enttäuscht», sagt Steinke, ohne bitter zu wirken. «In unserer Gesell-

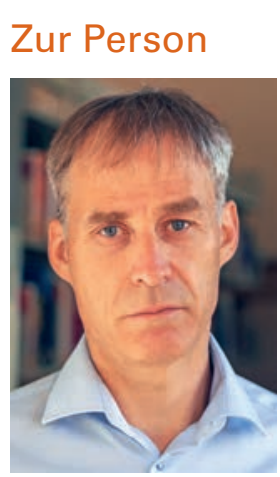

Dr. med. und Dr. phil. Hubert Steinke wurde 1966 in Niederwil (Solothurn) geboren. Er studierte an der Universität Bern zuerst Medizin, dann Kunstgeschichte, Geschichte und Philosophie. 1995 promovierte er in Bern zum Dr. med., 2003 in Oxford zum Dr. phil. Seit 2011 ist er an der Universität Bern Ordentlicher Professor für Medizingeschichte und Direktor des entsprechenden Instituts. Diesen Oktober hätte in Bern das Medizinmuseum eröffnet werden sollen, dessen Direktor er gewesen wäre. Im letzten Moment stoppte aber die Direktion des Inselspitals das Projekt. Seine Forschungsschwerpunkte sind die Medizinische Praxis des 18.-20. Jahrhundert, die Wissensproduktion im 18. Jahrhundert und das Thema "Medizin in Bern". Hubert Steinke ist verheiratet und Vater von zwei Kindern im Teenageralter. Er lebt mit seiner Familie in Bern.

schaft ist eine starke Tendenz zu einer Medikalisierung zu beobachten. Vieles, was früher 'normal' war, wird heute in einem medizinischen Licht gesehen: das Altwerden beispielsweise oder ein Zappelphilipp, dem heute Ritalin verschrieben wird. In einem solchen Umfeld müsste es doch Platz haben für einen Ort, wo ein öffentlicher Diskurs über Medizin angestossen werden kann. Das gehört auch zur gesellschaftlichen Verantwortung eines Universitätsspitals. So gesehen ist das Ende dieses Projekts eine verpasste Chance.» Immerhin passt das, was diesem Medizinhistoriker mit «seinem» Museum widerfahren ist, bestens zu einer anderen Entwicklung, die er bei der Medizin beobachtet: derjenigen einer zunehmenden Ökonomisierung.

"Ich weiss, dass sich gute Medizin immer nur am einzelnen Patienten manifestiert.»

\section{Und die Zukunft?}

Hubert Steinke hat zuerst Medizin studiert, dann Kunstgeschichte und Geschichte. Und dann wurde er Medizinhistoriker. Wobei er sich als Historiker mit medizinischem Hintergrund sieht, und nicht als Arzt mit historischem Background. Die Weichenstellung damals beschreibt er heute so: «Als Medizinstudent hatte ich das Gefühl, ersetzbar zu sein. Nach dem Motto: Wenn ich es nicht mache, macht es ein anderer.» Auch habe ihn die Metaebene, die intellektuelle Auseinandersetzung mit dem Fach, mehr interessiert als das individuelle Schicksal eines Patienten. Heute beurteile er 
zumindest den ersten Punkt etwas anders: «Ich weiss, dass sich gute Medizin immer nur am einzelnen Patienten manifestiert. Jeder Arzt, jede Ärztin kann den Unterschied machen.» Was die Metaebene in seiner heutigen Tätigkeit betrifft, sei es ihm wichtig, die Studenten an konkreten, praktischen Beispielen anzubinden, betont er.

Das tut er auch jetzt, bei der Frage nach der Zukunft der Medizin. Wenn er und eines seiner Kinder den Schnupfen habe, erhielten beide denselben Nasenspray, und die Dosierung sei auch gleich. «Solche undifferenzierten, standardisierten Therapien werden durch eine personalisierte Medizin abgelöst.» Und mit einem eher seltenen Schmunzeln fügt er bei: «In hundert Jahren wird man wohl über einiges lachen, was wir heute machen.»

\section{Forschen und fragen}

«Früher war es der Arzt und der Patient», stellt Steinke fest. Jetzt sei es ein ganzes System mit Krankenkassen, Patientenorganisationen, Pharmafirmen - und dem Staat. In solchen Prozessen des Wandels sei es Aufgabe eines Medizinhistorikers, Orientierungshilfen anzubieten, sagt Hubert Steinke. Die Grundlagen dazu findet er unter anderem in den Archiven, die ihm und seinen Studenten zur Verfügung stehen. Zurzeit leitet er ein Forschungsprojekt zu Albert Schweitzer. Aufgrund von Krankenakten und unzähligen Briefen will er herausfinden, welche Medizin dieser Pionier in Afrika betrieben hat. Das Archiv der Schweizerischen Akademie der Medizinischen Wissenschaften SAMW und bald auch jenes der FMH sind im Haus und gehören zu den Quellen des Instituts. Eines seiner geplanten Forschungsprojekte widmet sich denn auch der Entwicklung von ethischen Richtlinien in der Schweiz. "Grundsätzlich sind sie normativ, sagen also, wie man sich 'richtig' verhalten soll. Wir stellen fest: Die Gesellschaft wandelt sich, deshalb müssen sich auch die Richtlinien ändern.» Der kritische Blick auf dieses Thema führt zu einer kritischen Frage: «Es ist doch bemerkenswert, dass die SAMW eine private Stiftung aus dem medizinischen Umfeld ist - geben sich also die Mediziner ihre ethischen Richtlinien selber?»

\section{Sport - zur Freude}

Auf dem Gestell hinter dem Pult liegt neben alten Büchern ein Velohelm. "Das Schöne am Velofahren oder am Joggen ist für mich das Gefühl, meinen Körper zu spüren», kommentiert Hubert Steinke. Der Präventionsgedanke spiele für ihn beim Sport eigentlich keine Rolle. Das ist aus seiner Sicht auch so eine Entwicklung: «die Disziplinierung des Bürgers via schlechtes Gewissen». So gesehen sei er skeptisch gegenüber gewissen Gesundheitskampagnen. «Dort, wo es um die Gesundheit des Bürgers zum Wohle des Staates geht, sind sie aus meiner Sicht bisweilen fragwürdig.» Auch hier würden sich die Grundlagen übrigens immer wieder verändern. So sage eine neue Studie ja, Salzkonsum sei nicht so schädlich, wie man bisher angenommen habe. Wie umschreibt doch Hubert Steinke seine Aufgabe: Es gelte immer wieder kritisch hinzuschauen gerade in der Medizin. 